University of Wollongong

Research Online

Faculty of Engineering and Information

Faculty of Engineering and Information

Sciences - Papers: Part A

Sciences

$1-1-2012$

\title{
Wavelet transform based de-noising method for self mixing interferometry signals
}

\author{
Yuan Sun \\ University of Wollongong, ys857@uowmail.edu.au \\ Yanguang Yu \\ University of Wollongong, yanguang@uow.edu.au \\ Jiangtao Xi \\ University of Wollongong, jiangtao@uow.edu.au
}

Follow this and additional works at: https://ro.uow.edu.au/eispapers

Part of the Engineering Commons, and the Science and Technology Studies Commons

Research Online is the open access institutional repository for the University of Wollongong. For further information contact the UOW Library: research-pubs@uow.edu.au 


\title{
Wavelet transform based de-noising method for self mixing interferometry signals
}

\author{
Abstract \\ Self-mixing interferometry (SMI) signals are observed from a laser diode (LD) with optical feedbacks \\ induced by an external target. SMI signals carry information related to both of the target and parameters \\ of the LDs. However, the noise contained in SMI signals greatly degrades the applications of the SMI \\ systems. This paper proposes a wavelet transform based de-noising method which can effectively \\ eliminate noise while keeping an SMI waveform less changed. The proposed method is verified by both \\ simulations and experiments

\section{Keywords} \\ self, interferometry, signals, method, noising, mixing, de, transform, wavelet \\ Disciplines \\ Engineering | Science and Technology Studies

\section{Publication Details} \\ Y. Sun, Y. Yu \& J. Xi, "Wavelet transform based de-noising method for self mixing interferometry signals," in \\ 3rd Asia Pacific Optical Sensors Conference, 2012, pp. 83510G-1-83510G-7.
}




\title{
Wavelet Transform Based De-noising Method for Self Mixing Interferometry Signals
}

\author{
Yuan Sun, Yanguang Yu*, Jiangtao Xi \\ School of Electrical, Computer and Telecommunications Engineering, University of Wollongong, \\ Northfields Ave, Wollongong, NSW, 2522, Australia;
}

\begin{abstract}
Self-mixing interferometry (SMI) signals are observed from a laser diode (LD) with optical feedbacks induced by an external target. SMI signals carry information related to both of the target and parameters of the LDs. However, the noise contained in SMI signals greatly degrades the applications of the SMI systems. This paper proposes a wavelet transform based de-noising method which can effectively eliminate noise while keeping an SMI waveform less changed. The proposed method is verified by both simulations and experiments.
\end{abstract}

Keywords: self-mixing interferometry, de-noising, wavelet transform

\section{INTRODUCTION}

Optical feedback self-mixing interferometry (SMI) effect occurs when the laser beam reflected or backscattered from a distant target ahead of the laser diode (LD), re-enters the laser cavity, inducing the modulation of both the amplitude and the frequency of the lasing field. The modulated laser power, called SMI signal, is detected by a photodiode (PD). SMI signals carry information related to both of the target and parameters of the LDs. The basic structure of a SMI system is as shown as in Figure 1. This SMI technique has been extensively studied in last two decades ${ }^{1}$. Sensing applications based on this effect are extensively used to measure distances ${ }^{2}$, displacement and vibrations ${ }^{3-5}$, etc.

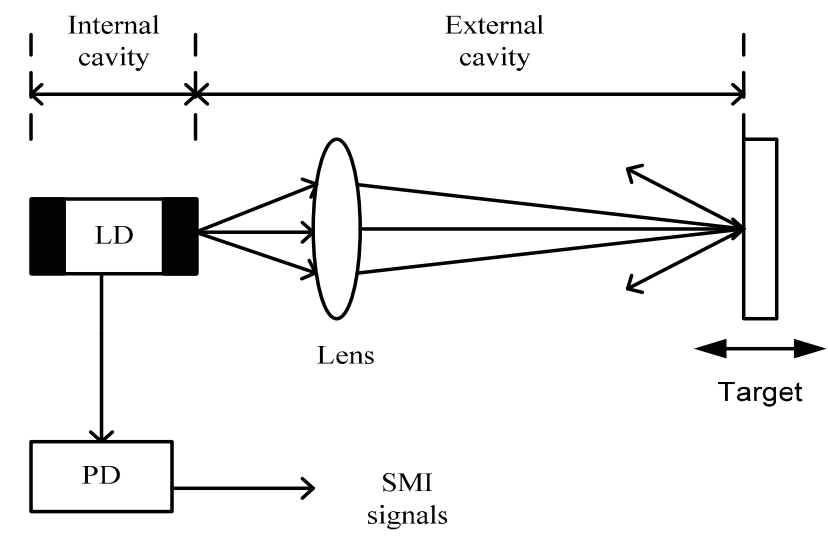

Figure 1. Basic structure of an SMI system

In order to retrieve the useful information carried by SMI signals for the sensing applications, high-quality SMI signals are required. In practice, SMI signals can be contaminated by many factors, such as ambient temperature, fluctuation in the LD driving source and other disturbances ${ }^{6}$. Mainly, two types of noises exist in a SMI signal, which are sparkle-like and high-frequency noise ${ }^{6}$. Figure 2 shows a SMI signal observed in our experimental set-up. The noise contained in SMI signals makes it difficult to locate those characteristic points, such as peaks and zero-crossing points which are often used to measure the parameters of $\mathrm{LDs}^{9,10}$. Therefore, de-nosing is a crucial step before the utilization of SMI signals. In order to reduce the noise of SMI signals, Yu et.al proposed a filtering method combined by median filter and band-pass filter with Kaiser window function ${ }^{6}$. However, the band-pass filter with Kaiser window function can change

Third Asia Pacific Optical Sensors Conference, edited by John Canning, Gangding Peng

Proc. of SPIE Vol. 8351, 83510G · (C) 2012 SPIE · CCC code: 0277-786X/12/\$18 · doi: 10.1117/12.915940

Proc. of SPIE Vol. $835183510 \mathrm{~B}-1$ 
the positions for some characteristic points such as the sharp transitions in a SMI waveform. A neural network interpolation technique for the noise elimination of SMI signals is presented by Lu et. $\mathrm{al}^{7}$. But this method only covered the weak and moderate optical feedback regimes and also had the same problems with the method ${ }^{6}$.

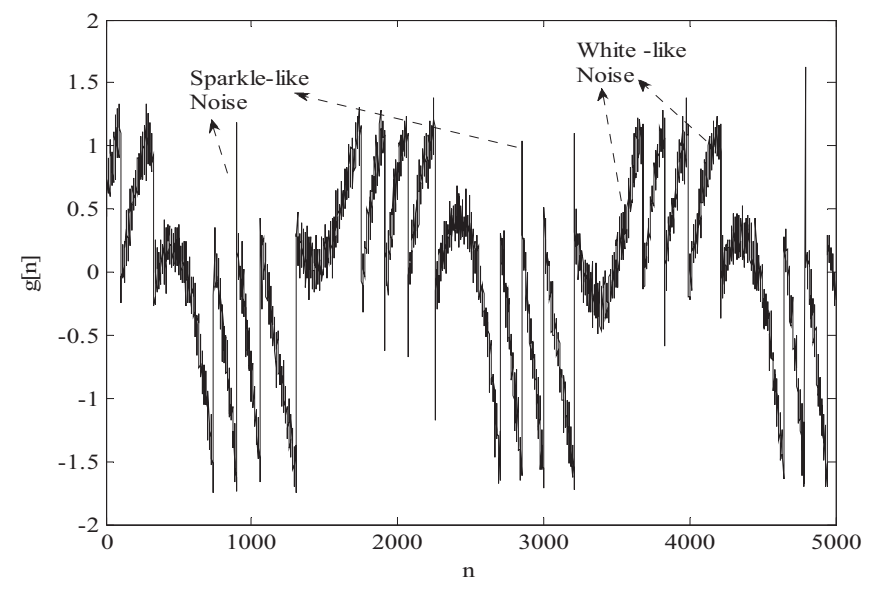

Figure 2. An experimental signal within sparkle-like noise and high frequency noise as labeled.

In this paper, we propose a novel de-noising method by a median filters and a wavelet transform based filter. The median filter is used to remove the sparkle-like noise, while wavelet transform based filter to reduce the high-frequency noise. The following part of this paper is organized as follows. Basic theory and features of SMI signals are demonstrated in Section II. Also in this section the noise feature and the objective of de-noising are also given. In section III the denoising method is proposed. Then both the simulation and the experiment results are presented in section IV. Conclusions are given in Section V.

\section{THEORY OF SEIF MIXING INTERFEROMETRIC SIGNAL}

The basic theoretical model for SMI systems is developed from Lang and Kobayashi ${ }^{8}$, and this model is described as the following equations ${ }^{3,4,9-11}$ :

$$
\begin{gathered}
\phi_{F}(n)=\phi_{0}(n)-C \sin \left[\phi_{F}(n)+\arctan (\alpha)\right] \\
P(n)=P_{0}[1+m \times g(n)] \\
g(n)=\cos \left(\phi_{F}(n)\right) \\
\phi_{0}(n)=4 \pi L(n) / \lambda_{0}
\end{gathered}
$$

In the above four equations, $n$ is the discrete time index. Equation (1) is the phase condition equation, which indicates the relationship between $\phi_{F}$ and $\phi_{0}$. These parameters are the external light phases of a LD with and without feedback, respectively, defined as $\phi_{F}=2 \pi v_{F} \tau$ and $\phi_{0}=2 \pi v_{0} \tau$, and where $v_{F}$ and $v_{0}$ represent the frequencies of the LD with and without feedback, respectively. $\tau=2 L / c$ is the round-trip time determined by the external cavity length $L$ and the light speed $c$. In Equation (2), $P(n)$ and $P_{0}$ denote the laser intensities with and without feedback, respectively. $m$ is the modulation parameter . $g(n)$ is called a SMI signal as defined by Equation (3) and it gives the impact of the external cavity length to the emitted power. There are also two important parameters in this model, linewidth enhance factor $(\alpha)$ and feedback level factor $(C)$, measurement methods of which have been studied ${ }^{9-11}$.

SMI signals have been intensively studied on in depth $^{4,12-13}$, which also have a fringe pattern similar to the conventional interference signals. The behavior of SLs can be classified into different feedback regimes when increasing feedback level ${ }^{14}$. In weak optical feedback regime, the waveforms of the SMI signals are sinusoid-like, while the signals exhibits hysteresis and the waveforms are saw-tooth shaped in both the moderate and strong feedback regimes. Lang-Kobayashi equations are widely accepted model for describing a SMI signal. Figure 3 shows a simulated SMI signal using Equations (1)-(4) with $C=2, \alpha=3$ based on this model. The positions of the characteristic points in the SMI signal waveform, including sharp transitions (denoted by $\mathrm{T}$ ), zero-crossing points (denoted by Z) and peak points (denoted by $\mathrm{P})$, carry information associated to the parameters of $\operatorname{LDs}^{9}$ and the moving information of the external target ${ }^{15}$. 
According to Equation (3), a normalized SMI signal varies in the range of $[-1,1]$. All those characteristic points in an SMI signal should be accurately selected so that a high-quality sensing can be achieved ${ }^{9}$.

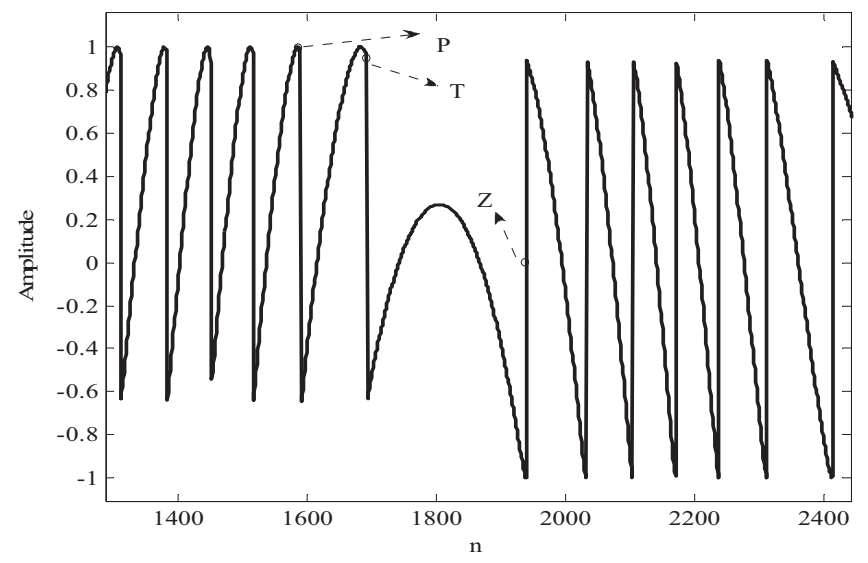

Figure 3. A simulated SMI signal in the moderate optical feedback regime: P: peak points; T: sharp transitions; Z: zerocrossing points. The parameters of this signal are listed: $C=2, \alpha=3$.

\section{DE-NOISING METHOD}

According to the noise feature and the waveform features of a SMI signal, we make the filtering requirements as below:

(a) Sparkle-like noise should be removed without changing of the locations of it and its surrounding points.

(b) The positions of sharp transitions should be kept unchanged after filtering.

(c) High-frequency noise should be reduced.

In 2007, Yu et.al indicated that usual moving average filters could not work well for SMI signals and presented a filtering method combined by a median filter and a band-pass filter based on Kaiser window function ${ }^{6}$. The median filter deals with sparkle-like noise very well but is poor for high-frequency noise. Kaiser window function based band-pass filter can improve the performance for high-frequency noise. Unfortunately, it can meanwhile change the waveform especially for the sharp transitions. To solve this problem, we propose to employ a wavelet transform based filter to replace the band-pass filter to preserve original waveform.

Wavelet transform is thought as "mathematical microscope" for its fine frequency property, which is appropriate for the processing of SMI signals. Wavelet transform based filter can reduce the noise effectively with little resolution loss and most sharp transitions can be preserved ${ }^{16-18}$. This provides a powerful tool for the processing of SMI signals. Wavelet transforms represent signals in multi-resolution, and decompose signals into different scales ${ }^{19-21}$. Sharp transitions are preserved and described very well in wavelet expansions ${ }^{21}$. Most noise power is restricted to small scales, so the filtering process at small scales decreases the noise preferentially ${ }^{22}$. Before applying the operations of wavelet transforms, the information of sharp transitions should be acquired and preserved during the filtration. In 1994, Xu et.al proposed an easy way to locate sharp transitions for images or signals using direct correlation between signals at different scales ${ }^{22}$. This method is efficient for most signals. However, for SMI signals, there is a better way to locate the transitions. The difference of the amplitude between two points in a sharp transition is large, so differential is employed to locate the position of the transitions.

The filtering method based on wavelet transform for SMI signals is as depicted in Figure 4 and the steps of this operation is demonstrated as below. 


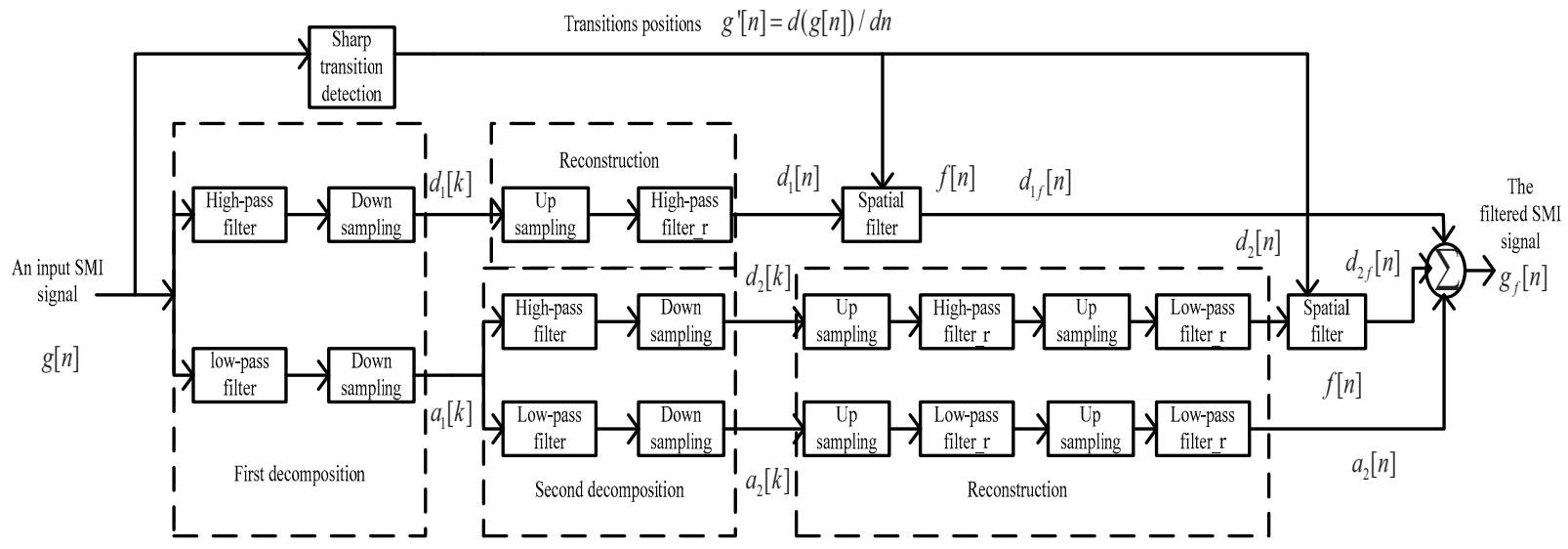

Figure 4. The flow chart of the wavelet transform based filtering process, where $g[n]:$ a sampled SMI signal; $g^{\prime}[n]$ : the differential of $g[n]$ which helps detecting the transitions; the blocks of high-pass and low-pass filters are the decomposition filters; the blocks of high-pass and low-pass filter_r are the reconstruction filters; the factor of upsampling and down-sampling is $2 ; d[k]$ : high frequency coefficients ; $a[k]$ : low frequency coefficients; $d[n]$ : reconstructed high frequency component; $a[n]$ : reconstructed high frequency component; $d[n]$ : filtered high frequency component; the filtered SMI signal.

Step1, discrete wavelet transform (DWT) is applied on a SMI signal to decompose it into different frequency contents. DWT is that the signal is passed through a series of low-pass filters and high-pass filters. The low-pass filters produce low frequency coefficients of the signal (denoted by $a[k]$ ), while the high-pass filters give high frequency coefficients ( denoted by $d[k]$ ). Down-sampling is needed after filtering the signal through the analysis filter bank because a single length input is being converted to a double length output. This operation is defined by the following equations ${ }^{23}$ :

$$
\begin{aligned}
& d[k]=\sum_{n=0}^{N-1} g[2 k-n] H[n] \\
& a[k]=\sum_{n=0}^{N-1} g[2 k-n] L[n]
\end{aligned}
$$

In this pair of equations, $N$ is the number of the filter taps and $g$ denotes the sampled self-mixing signal, $H[k]$ represents the high-pass filter and $L[k]$ indicates the low-pass filter. After the decomposition, $d[k]$ and $a[k]$ are obtained. Daubechies 4-tap (that is $\mathrm{N}=4$ ) wavelet is chosen as mother wavelet in this study because it has the best similarity with fringe shape of SMI signals, which results in the best performance. The corresponding FIR filters' coefficients are: lowpass filter $L[k]\{0.12940 .22410 .83650 .4830\}$; high-pass filter $H[k]\{-0.48300 .8365-0.2241-0.1294\}^{24}$.

Step 2, reconstruction is applied on each frequency component at deferent levels. The process is accomplished by passing each frequency component into the reconstruction filters and the up-sampling blocks. Then high frequency component $d[n]$ and low frequency content $a[n]$ are derived. The reconstruction FIR filters' coefficients are listed as

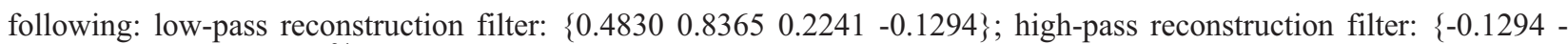
$0.22410 .8365-0.4830\}^{24}$. After the reconstruction, the reconstructed low frequency content is kept because most frequency content of the signal is included in this part, while the high frequency component is processed using the position information obtained in the next step. That is because the high frequency noise and the sharp transitions of the signal are contained in the high frequency content ${ }^{22}$.

Step 3, the key step of the processing is to locate the position of the transitions. Xu's method ${ }^{22}$ is easy and appropriate for most signals. But for SMI signal, taking differential operation of it is more precise and simpler. Then a spatially selective filter, $f[n]$, is applied on the reconstructed high frequency content with the positions information of the transitions; it spatially selects which part of the data (the transitions) to preserve and which part (noise) to eliminate. This spatial filter is illustrated as in Figure 5. Figure 5(a) gives a simulated noisy SMI signal ( $C=3, \alpha=3$, SNR $=20 \mathrm{~dB}$ ). The 
high frequency component after one level DWT is shown in Figure 5(b). Figure 5 (c) gives the result of the differential of the SMI signal and the spatial filter is demonstrated by the mask depending on the differential result in Figure 6 (d). After this spatial filtering, processed high frequency components $d_{f}[n]$ are obtained as in Figure 5(e).

Step 4, the processed high frequency components $d_{f}[n]$ are combined with the reconstructed low frequency component $a[n]$ to reconstruct the filtered signal. Since this filter reduces part of the high frequency part of the signal power, this filter can be seen as a low-frequency pass filter which is also passed by selective high frequency data.
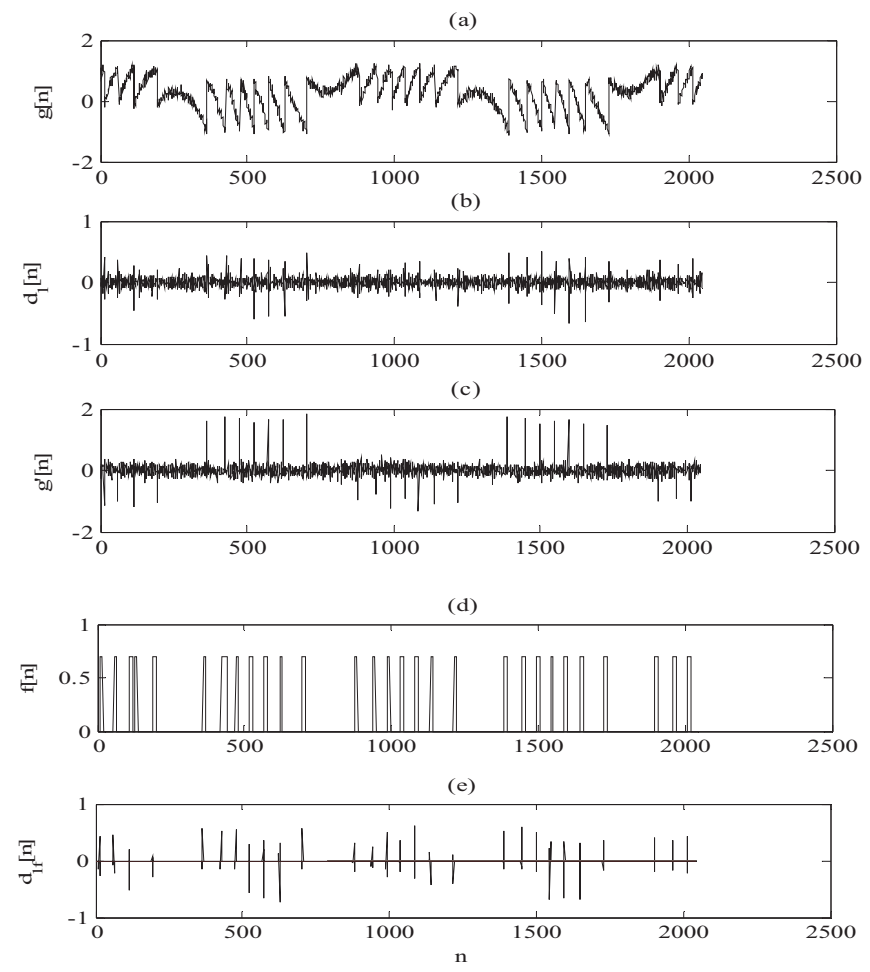

Figure 5. Illustration of the operation principle spatial filter: (a) a simulated SMI signal containing white noise of $20 \mathrm{~dB}$ ( $C=3$, $\alpha=3$ ); (b) decomposed high frequency component of signal $g[n]$ at first scale; (c) differential of signal $g[n]$; (d) a spatial filter; (e) the filtered high frequency component.

\section{SIMULATION AND EXPERIMENT VERIFICATION}

\subsection{Simulation verification}

A simulated SMI signal $g[n]$ is used to demonstrate this filtration technique graphically in Figure 6 . Several most important parameters are listed as below: linewidth enhancement factor $\alpha=3$, feedback level factor $C=3$, the frequency of the SMI signal is $71 \mathrm{~Hz}$ and the sampling frequency is $102.4 \mathrm{KHz}$. Gaussian white noise with SNR of $20 \mathrm{~dB}$ is added to evaluate this method. Figure 6(a) and (b) show the simulation signal without and with noise, respectively. After the wavelet filtering, most sharp edges are preserved and some of them are even being enhanced ${ }^{16-18}$. The latter parts need to be fixed. Thus, another median filter is utilized to process the data. The final filtered result is illustrated in Figure 6 (c), in which it can be seen the high frequency noise is reduced. Figure 6(d) shows that the enlarged transitions part before and after the filtering process. In this figure, it can be seen that the positions of sharp transitions are preserved. Thus, the noisy SMI is processed well. 


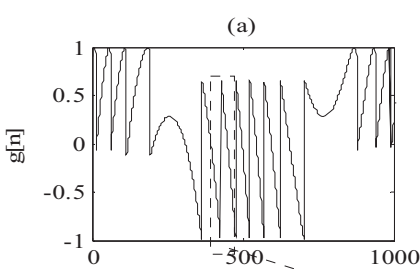

(c)

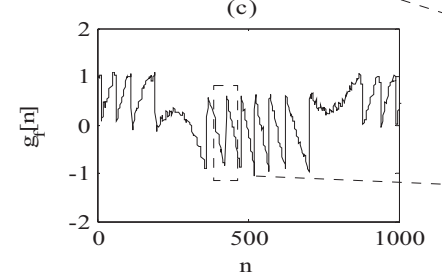

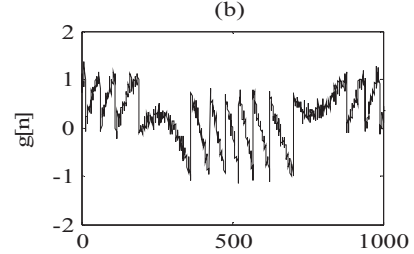

(d)

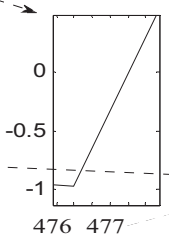

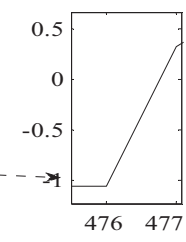

Figure 6. Filtering result for a simulated noisy SMI signal: (a) a simulated pure SMI signal; (b) the SMI signal with noise (SNR =20dB) (c) the filtered SMI signal; (d) an enlarged transitions parts before (left subfigure) and after (right subfigure) the filtering

\subsection{Experiment verification}

The proposed filtering method is also applied on experimental signals for verifying its correctness. The core part of the SMI experimental set-up is shown in Figure 1. The SMI signal is retrieved from the photo diode (PD) which is shown in Figure 7 (a). Figure 7 (b) illustrates the processed signal and an enlarged transition is shown in Figure 7(c). It can be seen clearly the sparkle-noise is removed and the high frequency noise is reduced. Also from the enlarged transition, it demonstrates that the sparkle-like noise is removed and the information of the transitions is preserved.
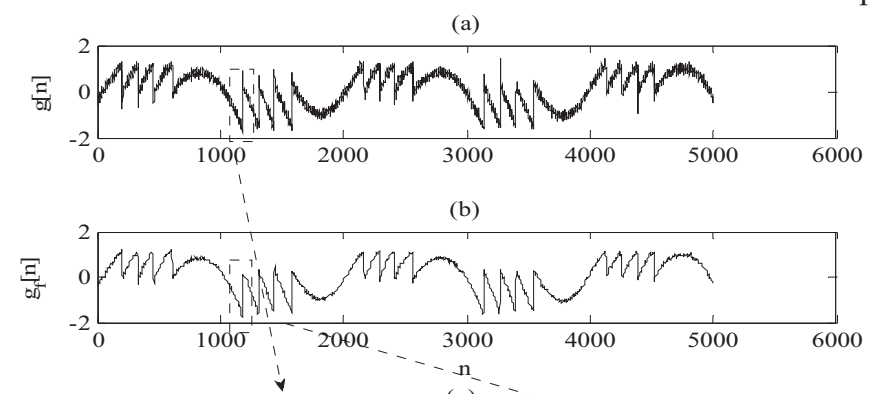

(c)

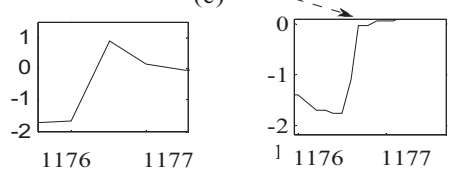

Figure 7. Filtering result for an experimental SMI signal (a) an original experimental SMI signal; (b) the filtered SMI signal (c) an enlarged transition before(left subfigure) and after(right subfigure) filtering

\section{CONCLUSION}

This paper describes a de-nosing method for SMI signals. Based on the noise features and characters of SMI signals, a wavelet transform based filtering method is proposed to apply on SMI signals. This method can greatly reduce the high frequency noise contained in SMI signals, meanwhile, sharp transitions on the waveform can be preserved very well. Both simulation and experiment are carried out to verify the performance of the filtering algorithm.

\section{ACKNOWLEDGMENT}

The work was supported by Australian Research Council on ARC Linkage International (LX0561454) and by National Natural Science Foundation of China (NSFC ID: 60871031 


\section{References}

[1] Bosch, T., “An Overview of Self-Mixing Sensing Applications,” Optoelectronic and Microelectronic Materials and Devices, 2004 Conference on , vol., no., pp.385-392, 8-8(2004).

[2] Bosch, T. and Lescure, M., "Selected Papers on Laser Distance Measurements," SPIE Milestone Series, vol. MS 115 (1995)

[3] Wang, W. M., Grattan, K. T. V., Palmer, A. W. and Boyle, W. J. O., "Self-mixing interference inside a singlemode diode laser for optical sensing applications," J. Lightwave Technol., vol. 12, pp. 1577-1587(1994).

[4] Donati, S., Giuliani, G. and Merlo, S., "Laser diode feedback interferometer for measurement of displacements without ambiguity," IEEE J. Quantum Electron., vol. 31, pp. 113-119 (1995).

[5] Bosch, T., Servagent, N. and Boyer, F., "Vibrations measurements with a self-mixing type laser displacement sensor for modal analysis," Instrumentation and Measurement Technology Conference, 1996. IMTC-96. Conference Proceedings. 'Quality Measurements: The Indispensable Bridge between Theory and Reality'., IEEE , vol.1, no., pp.648-653 vol.1(1996).

[6] Yu, Y., Xi, J. and Chicharo, J. F., "Improving the Performance in an Optical feedback Self-mixing Interferometry System using Digital Signal Pre-processing.” WISP, 1-6 (2007).

[7] Wei, L., Chicharo, J.F, Yu. Y., Xi, J., "Pre-Processing of Signals Observed from Laser Diode Self-mixing Intereferometries using Neural Networks," Intelligent Signal Processing, 2007. WISP 2007. IEEE International Symposium on , vol., no., pp.1-5, 3-5 (2007).

[8] Lang, R. and Kobayashi, K., "External optical feedback effects on semiconductor injection laser properties," IEEE J. Quantum Electron., vol. QE-16, pp. 347-355(1980).

[9] Yu, Y., Giuliani, G. and Donati, S., "Measurement of the linewidth enhancement factor of semiconductor lasers based on the optical feedback self-mixing effect," IEEE Photonics Technology Letters, vol. 16, pp.990992(2004).

[10]Xi, J., Yu, Y., Chicharo, J. F. and Bosch, T., "Estimating the parameters of semiconductor lasers based on weak optical feedback interferometry," IEEE Journal of Quantum Electronics, vol.41, No.8, pp.1058-1064(2005).

[11] Yu, Y., Xi, J., Chicharo, J. F. and Bosch, T., "Toward Automatic Measurement of the Linewidth Enhancement Factor Using Optical Feedback Self-Mixing Interferometry With Weak Optical Feedback," Quantum Electronics, IEEE Journal of 43, 527-534 (2007).

[12] K. Peterman,[ Laser diode modulation and noise], Dordrecht, The Netherlands: Kluwer(1988).

[13] Giuliani, G., Norgia, M., Donati, S. and Bosch, T., "Laser diode self-mixing technique for sensing applications," J .Opt. A: Pure Appl. Opt., vol. 4, No. 6, pp. S283-S294( 2002).

[14] Tkach, R. and Chraplyvy, A., "Regimes of feedback effects in 1.5-_mdistributed feedback lasers," IEEE Journal of Lightwave Technology, vol.4, no. 11, pp. 1655-1661(1986).

[15]Fan, Y., Yu, Y., Xi, J. and Chicharo, J. F., "Improving the measurement performance for a self-mixing interferometry-based displacement sensing system," Appl. Opt. 50, 5064-5072 (2011)

[16] Weaver, J. B., Xu, Y., Healy .Jr, D. M. and Cromwell, L. D., "Filtering noise from images with wavelet transforms," Magn. Reson. Med. vol. 21, no. 2, pp. 288-295(1991).

[17] Xu, Y., Lu, J., Healy, Jr., D. M. and J. B. Weaver, "Filtering MR images with wavelet transforms," in Proc. 10th Ann. Mtg. SMRM (San Francisco), p. 199(1999).

[18] J. Lu, Y. Xu, Weaver, J. B. and D. M. Healy, Jr., "Noise reduction by constrained reconstructions in the wavelet transform domain," in Proc. 7th IEEE SPS MDSP Workshop (Lake Placid, NY), p. 1.9(1991).

[19] Daubechies, I., "Orthonormal bases of compactly supported wavelets," Commun. Pure Appl. Math., vol. 41, pp. 909-996(1988).

[20] Mallat, S. G., “A theory for multiresolution signal decomposition: The wavelet representation,” IEEE Trans. Pa. Anal. Machine Zntell., vol. 11, no. 7, pp. 67\&693(1989).

[21] Mallat, S. G. and Zhong, S., “Complete signal representation with multiscale edges,' NYU Tech. Rep. No. 483(1989).

[22] Xu, Y., Weaver, J. B., Healy, Jr., D. M. and Lu, J., "Wavelet transform domain filters a spatially selective noise filtration technique," IEEE Trans Image Processing, 3(6) 747-758(1994).

[23] Strang, G. and Nguyen, T., "Wavelets and Filter Banks," Wellesley-Cambridge Press(1997).

[24] Daubechies, I., [Ten lectures on wavelets], CBMS, SIAM(1994). 